\title{
Educação na cultura digital: uma proposta de aprendizagem híbrida e
}

\author{
adaptativa
}

\section{Education in digital culture: a proposal for blended and adaptive learning}

\author{
Jéssica Andrade, Gilda Campos \\ Departamento de Educação - PUC Rio - Brasil
}

\begin{abstract}
Resumo
Este artigo apresenta o curso de especialização "Educação na Cultura Digital" - oferecido pelo Ministério da Educação à professores regentes da Educação Básica - a partir de uma análise do documento base que o fundamenta, realizada a partir da construção de categorias temáticas em sua interlocução com os dados da pesquisa TIC Educação (Cetic.br). Como resultado deste estudo identificamos que o curso possui em sua proposta indicativos que orientam uma prática docente na perspectiva de uma aprendizagem híbrida e adaptativa. Palavras-chave: formação continuada a distância, educação na cultura digital, aprendizagem híbrida, aprendizagem adaptativa.
\end{abstract}

\begin{abstract}
This article presents the specialization course "Education in Digital Culture" - offered by the Ministry of Education to the teachers responsible for Basic Education - based on an analysis of its guide's document, carried out from the construction of thematic categories in its interlocution with the data of the TIC Educação reasearch (Cetic.br). As a result of this study we have identified that the course has in its proposal indicatives that guide a teaching practice in the perspective of a hybrid and adaptive learning.

Keywords: distance continuing learning; education in digital culture, blended learning, adaptive learning.
\end{abstract}

\section{Introdução}

A definição de Cunha e Vilarinho (2009) sobre uma concepção emancipatória orientadora para os cursos de formação continuada a distância de professores encontra ressonância na proposta do curso de especialização "Educação na Cultura Digital", objeto de análise deste artigo. Segundo as autoras,

em uma perspectiva emancipatória da educação, os processos de formação continuada de professores apoiados na EAD precisam estar comprometidos com o desenvolvimento da capacidade de pensar criticamente associada a uma atitude coletivoreflexiva, que focalize a própria prática pedagógica. Quando as práticas formativas emancipatórias se articulam aos contextos de trabalho, mudanças significativas na educação podem ser observadas, colaborando para a (re)construção de uma sociedade mais democrática. (Cunha e Vilarinho, 2009, p.137)
O curso de especialização "Educação na Cultura Digital" tem $360 \mathrm{~h}$ de duração e destina-se aos educadores das redes de escolas públicas brasileiras com prioridade para os que estejam em exercício e nas funções de: Professores, Gestores e Formadores dos Núcleos de Tecnologia Estaduais e Municipais. Deve ser realizado por grupos, por escola, com pelo menos quatro professores, mais dois membros da equipe gestora e um formador. As agências formadoras são coordenadas nacionalmente pelo MEC e constituídas pelas Universidades e Secretarias de Educação Municipal e Estadual. Seu objetivo principal é formar educadores para integrar crítica e criativamente as tecnologias digitais de comunicação e informação (TDIC) aos currículos escolares. O curso é organizado em cinco dimensões de formação: pedagógica, tecnológica, comunicativa, ética e estratégica e sua metodologia está estruturada em três componentes principais: (1) o Plano de Ação Coletivo (PLAC) - responsável por formular, promover e executar propostas pedagógicas com uso de TDIC, (2) o Trabalho de Conclusão de Curso - um artigo reflexivo, inspirado nas ações das quais o cursista participou mais diretamente durante a realização do PLAC e das atividades desenvolvidas nos núcleos de estudos específicos (por disciplina), e (3) os Núcleos de Estudo - responsáveis por oportunizar o estudo, a análise crítica e o aprofundamento de temas relevantes à formação, do ponto de vista teórico e prático. Os recursos didáticos foram elaborado no modelo de autoria compartilhada entre um pesquisador, especialista na temática relativa ao Núcleo de Estudo, e um professor ou formador, que na sua prática esteja vivenciando as possibilidades pedagógicas estudadas. O sistema de acompanhamento visa avaliar e reorientar as estratégias de ensino e favorecer a aprendizagem do cursista. Um objetivo paralelo é reorganizar as formações iniciais de professores nos seus cursos de licenciatura, de modo a efetivamente preparar profissionais aptos a construir uma escola que promova "Educação na Cultura Digital" (Ramos et al., 2013).

Este artigo divide-se em quatro seções: na primeira é explicitada a metodologia utilizada. Na segunda são apresentadas breves considerações sobre os conceitos de aprendizagem híbrida e adaptativa. Na terceira, é feita uma análise de conteúdo, inspirada em Bardin, do 
documento base do curso "Educação na Cultura Digital" em sua interlocução com os dados da pesquisa TIC Educação (Cetic.br), procurando identificar se o mesmo possui em sua proposta indicativos que orientem uma prática docente na perspectiva de uma aprendizagem híbrida e adaptativa. Na quarta e última seção são apresentadas as considerações finais.

\section{Metodologia}

Para a análise do documento base do curso de especialização Educação na Cultura Digital, optou-se por uma análise de conteúdo (AC) por categorias temáticas inspirada em Bardin (1977, p.42) que define esta metodologia como "um conjunto de técnicas de análise das comunicações (...)" que tenta encontrar "uma série de significações que o codificador detecta por meio de indicadores que lhe estão ligados". Para proceder a classificação em categorias dos elementos encontrados no documento, é preciso identificar o que eles têm em comum, permitindo assim seu agrupamento. Em um primeiro momento foi feita a leitura do documento base que fundamenta o curso, procurando identificar termos ou expressões que indiquem uma orientação do curso para uma prática docente híbrida e adaptativa. Em um segundo momento os dados foram classificados em categorias de análise. E por último se procedeu à interpretação destas categorías em sua interlocução com os dados da pesquisa TIC e Educação (Cetic.br).

\section{Aprendizagem híbrida e adaptativa: uma alternativa de personalização do ensino}

Alguns questionamentos se colocam sobre o uso de tecnologia na educação na atualidade: Será realmente necessário que o professor leve diretamente os suportes e recursos tecnológicos para a sala de aula para que tenhamos o efetivo uso das Tecnologias da Informação e Comunicação (TIC) nas escolas/universidades? Será que um caminho possível para a efetiva aplicação das tecnologias à educação pode prescindir, porém sem substituir, da utilização do espaço da sala de aula e até mesmo da escola/universidade? Quais são as propostas existentes no cenário atual?

Os MOOCS (Massive Open Online Courses) são uma das mais claras manifestações das novas relações que se estabelecem entre as TIC e a educação. Cursos universitários online, gratuitos e disponíveis a qualquer indivíduo com acesso à internet, com a possibilidade de certificação após a conclusão dos mesmos, cada vez mais parecem tentar romper a barreira entre ensino presencial e a distância, trazendo à tona com mais força - na medida em que estes cursos são oferecidos por universidades renomadas e de prestígio internacional - um novo formato ou uma nova proposta para o processo de ensinoaprendizagem: a aprendizagem híbrida, onde parte do processo ocorre em ambientes virtuais de aprendizagem e parte em salas de aula físicas, como no modelo tradicional de ensino.

Atendendo a uma perspectiva híbrida da aprendizagem se constitui a aprendizagem adaptativa, cujos elementos ensejados em sua lógica são a autonomia, o protagonismo do aluno, a personalização do ensino e o acompanhamento e avaliação contínua da aprendizagem.
Estas não são ideias novas e também estão presentes na educação tradicional exclusivamente presencial. A principal diferença reside no fato de reconhecer na tecnologia o potencial de expansão das formas e lugares de aprendizagem dos alunos, constituindo-se em uma via paralela e integradora, não substitutiva e nem tampouco de caráter simplesmente complementar ao ensino presencial, para alcance dos objetivos de aprendizagem.

A personalização do ensino, possibilitada pela construção de algoritmos - sequência lógica, finita e definida de instruções que devem ser seguidas para resolver um problema ou executar uma tarefa -, configura-se como elemento central da aprendizagem adaptativa, estimulando o protagonismo do aluno, a construção de sua autonomia e subsidiando um processo de avaliação contínua da aprendizagem. As chamadas plataformas de aprendizagem adaptativa, tem como objetivo não somente permear o processo de ensinoaprendizagem, mas principalmente oferecer subsídios de forma rápida e eficaz a tudo o que envolve este processo. Elas são pensadas em sua constituição, a partir das diferentes formas através das quais um aluno pode aprender, levando em consideração os diferentes comportamentos e reações possíveis aos conteúdos apresentados na plataforma. Bechara e Haguenauer (2010) auxiliam a compreensão da noção de aprendizagem adaptativa ao apresentar o conceito de estilos cognitivos ou de aprendizagem, que se refere a forma como as informações são recebidas, processadas e usadas pelo indivíduo.

O intuito destas plataformas é permitir que (i) os estudantes possam identificar claramente seus pontos de fragilidade na compreensão de diferentes conteúdos - ao realizar tarefas no ambiente virtual de aprendizagem, o retorno pode ser dado imediatamente, gerando recomendação de novas atividades que visam corrigir estas fragilidades; (ii) os pais possam acompanhar diretamente a evolução dos filhos na escola; (iii) os professores possam monitorar e intervir de forma personalizada e contínua na aprendizagem dos alunos; (iv) os diretores tenham a possibilidade de saber exatamente como a escola está caminhando.

\section{O curso de especialização "Educação na Cultura Digital" e sua interface com a proposta de aprendizagem híbrida e adaptativa}

A partir da leitura do documento base que fundamenta o curso, foram identificados termos ou expressões que indicam os pressupostos básicos do mesmo e que estão em consonância com uma prática docente híbrida e adaptativa. Estes dados foram classificados em categorias de análise (Quadro 1), procedendo em seguida a sua intepretação. 
Quadro 1

Categorias de análise do documento base do curso de especialização "Educação na Cultura Digital"

\begin{tabular}{|c|c|}
\hline $\begin{array}{l}\text { Categorias } \\
\text { de Análise }\end{array}$ & Definição no Documento Base \\
\hline $\begin{array}{l}\text { Cultura } \\
\text { Digital }\end{array}$ & $\begin{array}{l}\text { Não está consolidado e aproxima-se de } \\
\text { outros como sociedade da informação, } \\
\text { cibercultura, revolução digital, era digital. }\end{array}$ \\
\hline TIC & $\begin{array}{l}\text { Ferramentas estruturantes da atividade } \\
\text { pedagógica; Tecnologias da Cognição; } \\
\text { Tecnologias da Inteligência; Instrumentos } \\
\text { de suporte ao pensamento reflexivo e de } \\
\text { produção cultural; Geram novos conceitos, } \\
\text { métodos e processos. }\end{array}$ \\
\hline Currículo & $\begin{array}{l}\text { É processo, envolvendo uma multiplicidade } \\
\text { de relações, abertas ou tácitas, em diversos } \\
\text { âmbitos, que vão da prescrição à ação, das } \\
\text { decisões administrativas às práticas } \\
\text { pedagógicas. }\end{array}$ \\
\hline $\begin{array}{l}\text { Web } \\
\text { currículo }\end{array}$ & $\begin{array}{l}\text { Currículo envolve tanto propiciar ao aluno } \\
\text { a compreensão de seu ambiente cotidiano } \\
\text { como comprometer-se com sua } \\
\text { transformação; Sujeitos da aprendizagem } \\
\text { devem ser produtores e autores de } \\
\text { conteúdos digitais, nas várias mídias e } \\
\text { linguagens digitais disponíveis. }\end{array}$ \\
\hline Meto & $\begin{array}{l}\text { Articulação constante entre teoria e prática; } \\
\text { O professor deve produzir seus próprios } \\
\text { projetos e conteúdos, individual e/ou } \\
\text { coletivamente, e incentivar a produção dos } \\
\text { alunos nas diferentes mídias, de forma } \\
\text { articulada à proposta pedagógica e aos } \\
\text { currículos escolares; Formação do professor } \\
\text { baseada nos princípios da continuidade, } \\
\text { flexibilidade, autonomia e ação coletiva; } \\
\text { Investigação e pesquisa como princípio } \\
\text { pedagógico. }\end{array}$ \\
\hline $\begin{array}{l}\text { Inovação } \\
\text { tecnológica }\end{array}$ & $\begin{array}{l}\text { Mudanças nos métodos, técnicas, } \\
\text { modalidade e materiais de ensino. }\end{array}$ \\
\hline $\begin{array}{l}\text { Coletividade, } \\
\text { Cooperação, } \\
\text { Colaboração }\end{array}$ & $\begin{array}{l}\text { Ênfase na produção coletiva de } \\
\text { conhecimento; Estímulo para a criação de } \\
\text { redes e comunidades virtuais de } \\
\text { aprendizagem e prática que promovam a } \\
\text { partilha, a troca e a veiculação de conteúdos } \\
\text { educacionais. }\end{array}$ \\
\hline Autonomia & $\begin{array}{l}\text { Reconhecimento e promoção do papel da } \\
\text { escola como agência formadora; Promoção } \\
\text { de uma atuação crítica e criativa de caráter } \\
\text { emancipatório; Autoria. }\end{array}$ \\
\hline
\end{tabular}

O curso de especialização "Educação na Cultura Digital" é oferecido no âmbito do PROINFO Integrado e atende a uma demanda formada por uma alteração do perfil de uso e de acesso às tecnologias nas comunidades escolares após a realização de diversos programas de inclusão digital. Para os autores do curso, assim se constituíram as bases para a ampliação da cultura digital no país, entendida como a cultura da contemporaneidade e definida como uma mudança cultural, em uma época marcada pelas relações humanas com forte mediação das tecnologias e comunicações digitais. Mesmo entendendo que o processo de alfabetização tecnológica está dado, o curso pressupõe o uso de estratégias iniciais flexíveis para atender os diferentes perfis de competência e fluência digital dos educadores.
Há uma discussão interessante sobre inserção, uso e incorporação das TIC na educação. As séries históricas da pesquisa TIC Educação (CETIC, 2010 a 2014) revelam que os professores tem se utilizado cada vez mais das tecnologias no âmbito profissional (Figuras 1), no que concerne à pesquisa e preparação de aulas (Figura 2), o processo de inserção e uso, portanto, está dado. Entretanto a incorporação das TIC em sala de aula, traduzida em "uma mudança metodológica nas formas de ensinar e aprender" (Ramos et al., 2013, p.8) ainda está em processo (Figura 3).

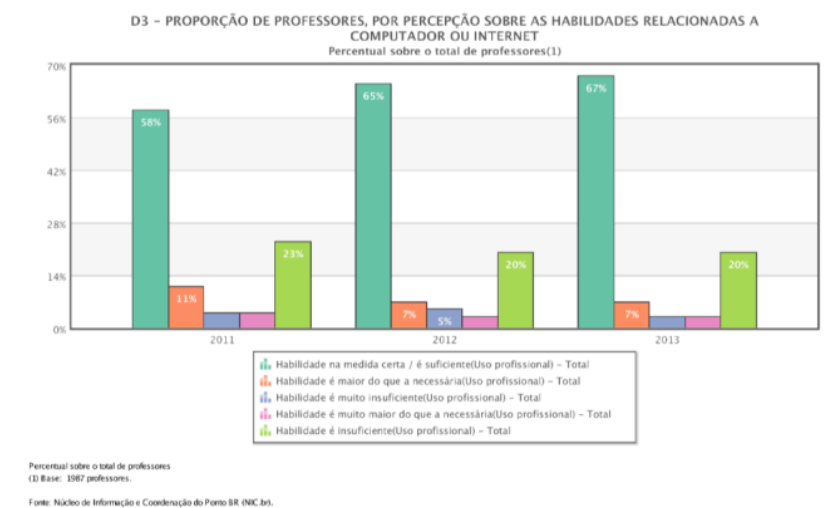

Figura 1-Uso Profissional de Computador e Internet. Fonte: Pesquisa TIC Educação -CETIC, Série Histórica 2011-2013.

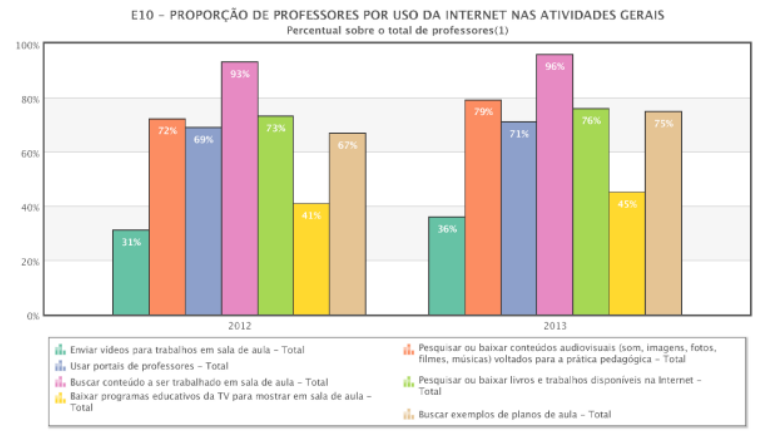

Figura 2 - Uso da Internet em Pesquisa e Preparação de Aulas. Fonte: Pesquisa TIC Educação -CETIC, Série Histórica 2012-2013.

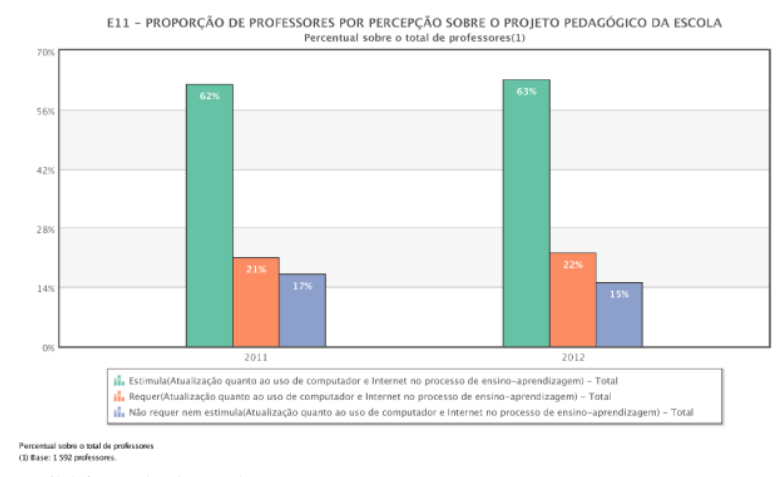

Figura 3 -Uso de Computador e Internet no Projeto Pedagógico da Escola. Fonte: Pesquisa TIC Educação CETIC, Série Histórica 2011-2012. 
É feita uma crítica em relação à lógica de consumo que consubstancia a maioria dos cursos de formação em tecnologias na educação, incluindo os ofertados pelo Proinfo Integrado, onde as experiências da escola não são levadas em consideração, destituindo o professor do seu papel de protagonista no processo de ensinoaprendizagem. Outra crítica consiste na identificação do caráter ora instrumental - aprendizado das ferramentas tecnológicas -, ora reflexivo - análise teórico-pedagógica -, destes cursos, sem a sua necessária integração à prática escolar. Nesse sentido, o objetivo do curso é "pensar a formação de educadores e professores menos em termos de conteúdos e pacotes prontos e mais no fortalecimento de sujeitos capazes de pensar e promover a sua formação, com foco numa metodologia que pensa a escola a partir dela mesma" (RAMOS et al., 2013, p.8).

A inovação na educação com o uso das TIC concebidas como ferramentas estruturantes da atividade pedagógica - é compreendida a partir de três dimensões (i) uso de novos materiais e tecnologias curriculares; (ii) uso de novos enfoques de ensino; (iii) alteração de crenças e pressupostos pedagógicos em relação às novas políticas ou programas educacionais, que devem ser propostas articuladamente, favorecendo uma "formação que transcenda a oferta de conteúdos e promova a formação para uma mudança metodológica e um trabalho coletivo e colaborativo na escola" (RAMOS et al., 2013, p. 9). Portanto, a introdução das tecnologias na escola não deve se configurar como um objetivo em si mesmo, mas sim permear todos os processos e objetivos educacionais, construindo um significado cultural para o seu uso. A ênfase na coletividade, baseada nos princípios de cooperação e colaboração, é consubstanciada pelos dados da pesquisa TIC Educação (CETIC, 2010 a 2013), onde a colaboração entre pares aparece como a principal forma de apoio ao uso das TIC na escola (Figura 4).

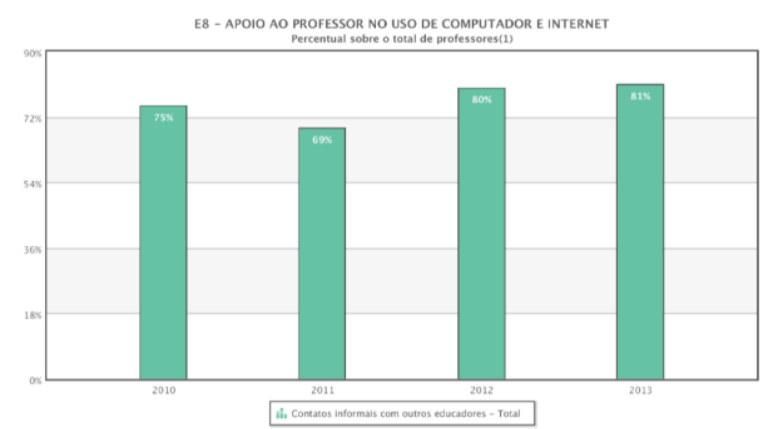

Figura 4 - Colaboração entre Pares. Fonte: Pesquisa TIC Educação -CETIC, Série Histórica 2010-2013.

Dados da última pesquisa TIC e Educação do Cetic.br (2015), apontam que uma boa parte dos cursos de licenciaturas ainda não inseriram em seu currículo disciplinas que ofereçam subísídios para uma prática pedagógica que incorpore as TIC, na medida que apenas $39 \%$ dos respondentes tiveram alguma disciplina específica na graduação/licenciatura sobre como usar computador e internet em atividades com alunos (Figura 5). Os professores uscam sozinhos (91\% dos respondentes) se atualizar quanto ao uso de computador e internet (Figura 6), o que aponta o descompasso entre as demandas da sala de aula e o currículo das licenciaturas.

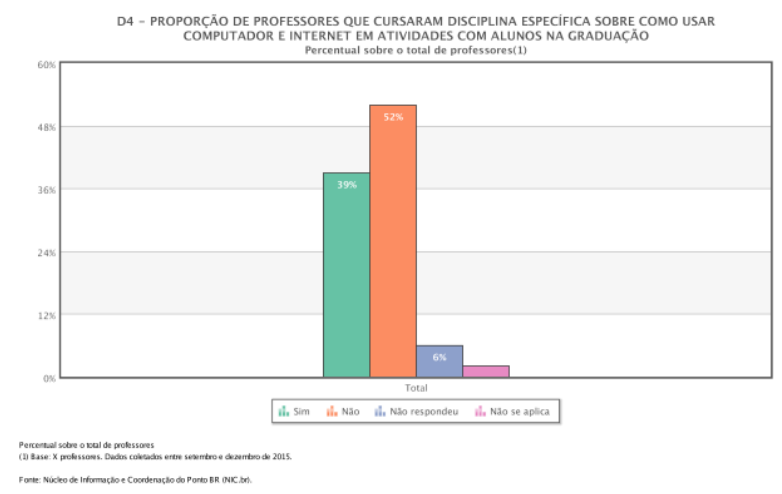

Figura 5 - Professores que cursaram disiplina específica, na graduação, sobre uso de computador e internet em atividades com alunos. Fonte: Pesquisa TIC Educação CETIC, 2015.

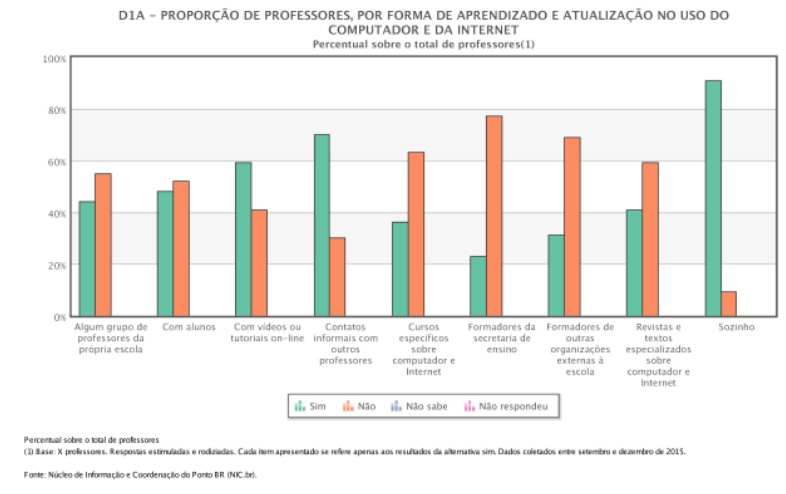

Figura 6 - Forma de aprendizado e atualização no uso de computador e internet. Fonte: Pesquisa TIC Educação CETIC, 2015.

Os principais indicativos de que existe uma confluência entre a proposta do curso e a orientação para uma aprendizagem híbrida e adaptativa se encontram nos seguintes aspectos, listados abaixo:

- A proposta de uma arquitetura pedagógica organizada em núcleos de estudo modulares - podendo estes núcleos serem cursados em diferentes composições - mantendo interdependência e organicidade entre si a partir de um projeto comum, permitindo, desse modo, que cada escola faça seu próprio projeto de formação diferenciado que atenda ao perfil individual dos seus profissionais e às demandas de sua realidade (Ramos et al, 2013, p.14).

- Construção de um Projeto Político Pedagógico (PPP) que pressuponha arranjos mais criativos e flexíveis de tempo-espaço (Ramos et al, 2013, p.17).

- O planejamento não pode ser completamente prédefinido, uma vez que se esteja transformando a prática em algo que ainda não existe, e que depende do significado que os participantes irão criar, Ao invés disso, deve ser desenhado um ambiente que evoque e facilite o surgimento dos diversos tipos de práticas preferidas (Cavallo, 2000 apud Ramos et al, 2013, p.15). 
- Através das "tecnologias da inteligência” é possível organizar toda uma estrutura de "inteligência coletiva" que potencializa nossa capacidade de produzir conhecimento em rede. Com seu potencial expressivo, as TIC permitem atingir um elevado padrão de comunicação com sensibilidade, não só ao contexto geográfico, mas também aos caminhos virtuais trilhados durante a aprendizagem. (Ramos et al, 2013, p.12).

- Pode-se afirmar que a proposta de aprendizagem híbrida e adaptativa se localiza no contexto do Modo 2 de produção do conhecimento proposto por Gibbons et al. (1994), e está presumida no curso "Educação na Cultura Digital", na medida em que se caracteriza pelo fluxo constante entre o teórico e o prático - o aluno acessa e estuda os conteúdos online, faz exercícios, recebe o retorno sobre os mesmos ainda na plataforma, discute presencialmente com o professor e seus pares sobre o que aprendeu ou deixou de aprender, partindo assim para a aquisição de novos conteúdos. O conhecimento no Modo 2 - sempre mediado pelas TIC - é resultado de um fenômeno coletivo, abrindo espaço para o entendimento de que a adaptação e personalização do ensino não são sinônimo de individualização absoluta do processo de aprendizagem, nem de anulação do coletivo no referido processo.

\section{Considerações finais}

Com um referencial teórico pautado em Piaget, Lèvy e Almeida e Silva, o curso cria contornos específicos para uma educação na cultura digital: uma educação construtivista, onde as tecnologias mobilizam a produção do conhecimento em rede, estruturando assim um web currículo.

Comparando a proposta do curso "Educação na Cultura Digital" com a do curso "Tecnologias na Educação: Ensinando e Aprendendo com as TIC", também oferecido no âmbito do Proinfo Integrado, pode-se identificar, ao menos no campo da proposição, o salto qualitativo defendido pelos autores, traduzido em uma "formação que transcenda a oferta de conteúdos e promova a formação para uma mudança metodológica e um trabalho coletivo e colaborativo na escola". No curso "Tecnologias na Educação" a articulação entre currículo e tecnologias é tema apenas de uma unidade do curso e proposta a partir da adoção de uma metodologia específica, a pedagogia de projetos (Andrade e Campos, 2015). Neste sentido percebe-se um avanço no intuito de construir um web currículo.

A defesa de ambientes escolares com mais mobilidade - tecnológica e de pessoas - faz avançar na perspectiva de uma aprendizagem para além dos muros da escola, onde o presencial e on-line possam se revezar e completar. Entretanto alguns entraves se colocam, na medida em que "a inserção das tecnologias digitais no trabalho pedagógico precisa ser refletida no Projeto Político Pedagógico das escolas e das redes de Ensino, uma vez que demandam reorganizações curriculares, mudanças estruturais, tempo coletivo para estudo, planejamento e avaliação e estabilidade do quadro de profissionais" (Ramos et al., 2013, p. 14).

\section{Referências}

Andrade, J.Z. \& Campos, G.H.B. (2015) TIC e Currículo: Uma Conjugação Possível. Porto Alegre, RENOTE, vol 13, n.2, p.1-10. http://seer.ufrgs.br/index.php/renote/article/view/6143 $0 / 36322$

Bardin, L. (1977) Análise de conteúdo. Lisboa: Edições 70.

Bechara, J. J. B. \& Haguenauer, C.J. (2010). Por uma aprendizagem adaptativa baseada na plataforma Moodle. Rio de Janeiro, EducaOnline, vol. 4, n ${ }^{\circ}$, p.110.

http://www.latec.ufrj.br/revistas/index.php?journal=e ducaonline \&page $=$ article $\&$ op $=$ view $\&$ path $\% 5 \mathrm{~B} \% 5 \mathrm{D}=$ 95\&path $\% 5 \mathrm{~B} \% 5 \mathrm{D}=130$

CETIC. (2010 a 2015) TIC e Educação. http://data.cetic.br/cetic/, acessado em 07/12/15.

Cunha, M.L. e Vilarinho, L.R.G. (2009) Concepção emancipatória: uma orientação na formação continuada a distância de professores. Curitiba, Revista Diálogo Educacional, v. 9, n. 26, p. 133-148. https://periodicos.pucpr.br/index.php/dialogoeducacio nal/article/view/3688/3604

Gibbons, M., Lomoges, C., Nowotny, H., Schwartzman, S., Scott, P., Trow, M. (1994) The new production of knowledge: the dynamics of science and research in contemporary societies. England: Sage.

Ramos, E.M.F., Cerny, R.Z., Cavellucci, L.C.B, Silva, M.R., Búrigo, C.c.D., Hassan, E.B.(2013) Curso de especialização em educação na cultura digital: Documento Base. Brasília: MEC. http://educacaonaculturadigital.mec.gov.br/downloads /documento-base.pdf 